\title{
Rhithrogena ryszardi n. sp., Ephéméroptère nouveau du Moyen Atlas (Maroc) et redescription de $R h$. soteria Navás, 1917 (Heptageniidae)
}

\author{
A.G.B. Thomas ${ }^{1}$ \\ B. Vitte 2 \\ T. Soldán ${ }^{3}$
}

Mots clés : Ephéméroptères, Heptageniidae, espèce nouvelle, Maroc, redescription, holotype, Espagne.

Répartition géographique comparée des trois espèces de Rhithrogena du groupe germanica sensu stricto de l'Ouest de l'Europe et de l'Afrique du Nord. Redescription de l'holotype de $R h$. soteria Navás, 1917 (imago ó, terra typica: l'Espagne) et description de Rh. ryszardi n. sp. (imago o) provenant du Moyen Atlas. Cette dernière est nettement moins orophile que les trois autres espèces de ce genre déjà signalées du Maroc.

Rhithrogena riszardi n. sp., a new Ephemeroptera species from the middle Atlas (Morocco) and a redescription of Rh. soteria Navás, 1917 (Heptageniidae).

Keywords : Ephemeroptera, Heptageniidae, new species, Morocco, redescription, holotype, Spain.

The geographical distribution was compared for three species of Rhithrogena of the group germanica sensu stricto from Western Europe and North Africa. The holotype of $R h$. soteria Navas, 1917 (male imago, terra typica : Spain) is redescribed and $R h$. ryszardi $n$. sp. (male imago) coming from the middle Atlas is described. The latter species is clearly less orophile than the three other species of this genus already recorded from Morocco.

\section{Les Rhithrogena du groupe germa- nica sensu stricto}

Le groupe germanica sensu stricto peut être défini sommairement par les caractères suivants :

- similitude morphologique des genitalia o (en particulier, les lobes péniens sont longs, subrectilignes, avec un apex massif et peu ou pas armé) ; titillateurs très larges et multidentés;

- grande taille (longueur de l'aile antérieure de l'ordre de 11 à $15 \mathrm{~mm}$ pour le ơ, soit le double de celle de $R h$. gorrizi par exemple, bien moins étroitement apparentée ;

1. Laboratoire d'Hydrobiologie, UA 695 du C.N.R.S., Université P. Sabatier, 118, route de Narbonne, 31062 Toulouse Cedex, France.

2. Laboratoire de Faunistique, Département de Biologie, Faculté des Sciences, Université S Med Ben Abdallah, B.P. 1796 Atlas, Fes, Maroc.

3. Ceskoslovenska akademie ved, Entomologicky ustav, Branisovska 31, 37005 Ceske Budejovice, Tchécoslovaquie.
- période de vol printanière.

Trois espèces actuellement connues, probablement vicariantes, constituent ce groupe : $R \boldsymbol{h}$. germanica Eaton, $R h$. soteria Navás et $R h$. ryszardi n. sp. Leur répartition dans le Sud-Ouest de l'Europe et l'Afrique du Nord est schématisée sur la carte $t$. Elles sont nettement moins affines de deux autres espèces, espagnoles et très proches l'une de l'autre : $R h$. gorrizi Navás, 1913 (voir Thomas et Sartori, 1985) signalée de l'Ebre à Saragosse et $R h$. thomasi Alba-Tercedor et Sowa, 1986, décrite à partir d'un seul spécimen provenant des environs de Valence.

\section{Rhithrogena germanica Eaton, 1885}

Descriptions de référence : Eaton(t.c.) et surtout Sowa (1971) qui, le premier, a clairement caractérisé l'espèce.

Localité typique: le Rhin, près de Laufenburg (Suisse). 


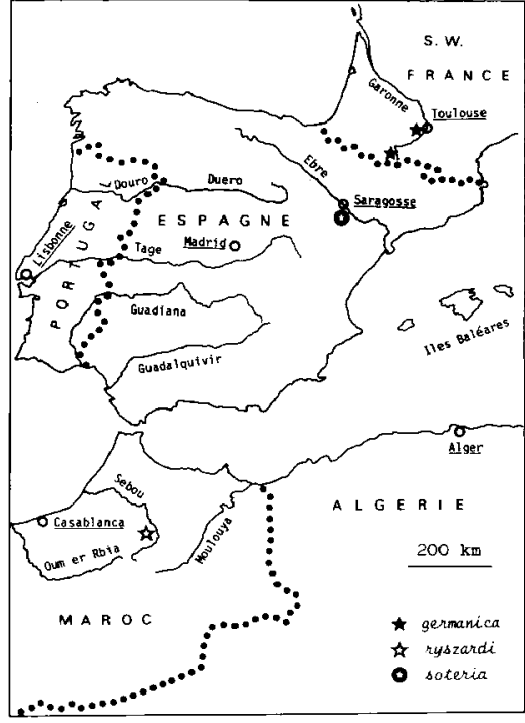

Carte 1. Répartition des espèces de Rhithrogena du groupe germanica sensu stricto en Europe du Sud-Ouest et en Afrique du Nord.

L'aire de répartition (Puthz 1978) recouvie les régions $2,9,10,13,14,16$ et 18 de la Limnofauna Europaea, c'est-à-dire environ du $43^{\mathrm{e}}$ au $59^{\mathrm{e}}$ degrés de latitude Nord, avec, pour limites :

- au Nord : la Grande Bretagne (Highlands : Macan 1979) et le Danernark (Sowa, t.c.).

- au Sud : les environs de Toulouse (Thomas) et les Pyrénées en dessous de $1000 \mathrm{~m}$ (Despax, 1949). L'espèce ne paraît pas traverser la chaîne pyrénéenne: elle n'a en effet pas été signalée dans la péninsule ibérique (Alba-Tercedor 1981) ni en Andorre (Puig 1979) - cette dernière étude toutefois effectuée seulement sur du matériel larvaire. Elle est aussi absente d'Italie, même septentrionale (Belfiore 1983). Nos citations de cette espèce du SudQuest de la France sont donc les plus méridionales connues.
$R h$. germanica est caractéristique des grands cours d'eau rapides, peu pollués, ce qui a deux conséquences :

- la restriction de son aire de répartition aux altitudes modérées ou basses (Sowa 1975).

- et actuellement sa disparition progressive des grandes rivières à cause des perturbations d'origine humaine (il est à peu près certain qu'elle ne se trouve plus dans le Rhin par exemple).

Sa période de vol s'étend du début mars à la fin mai, tout au plus aux premiers jours de juin (Despax, Sowa, Macan t.c., présentes données).

\section{Matériel examiné}

Suisse : 1 imago (i.) ơ piquée à sec (l'Aar à Belp, 2-IV-20) dans les collections du Museum de Berne.

Ecosse : 1 i. ơ (la Tweed à Innerlesthen, 7-15-IV-1931, M.E. Mosely leg.) dans la collection Thomas.

France : Département de la Haute-Garonne : 4 i. $\sigma$ obtenues par élevage (la Garonne en amont de Toulouse, 140 m, 25-IV-1973, Coll. A. Thomas).

Département de l'Ariège : 1 i. $\sigma$ (le Lez à Moulis, $430 \mathrm{~m}$, 22-III-1964, C. Berthélemy leg., R. Sowa dét.) dans la collection Sowa à Cracovie.

Despax (1949) a également cité cette espèce (s.n. haarupi = germanica - voir Sowa 1971 - détermination confirmée par M.E. Mosely) de la Garonne près de Luchon, vers $500 \mathrm{~m}(11.12 \cdot$ IV-1939:2 i. $\sigma)$ et de son affluent la Pique, vers $900 \mathrm{~m}$ (12-III-1939: 1 i. o capturée sur la neige).

\section{Rhithrogena soteria Navás, 1917}

Description originale sur un seul spécimen (Navás t.c.). Validité de l'espèce confirmée par Thomas (1968). A notre connaissance, $R h$. soteria n'a pas été retrouvée depuis Navás et seul l'holotype peut servir de référence.

\section{Matériel examiné}

1 i. O piquée à sec qui, bien que ne portant pas l'étiquette rouge habituelle de Navás ("Typus "), parait ne pouvoir être que l'holotype (Zaragoza, 29-1II-17).

Etat général du spécimen plutôt bon et identification non douteuse. Il manque une patte antérieure et les cerques. Le thorax est fissuré sur presque toute sa longueur, près du plan sagittal.

Ce type est déposé au Musée Zoologique de Barcelone où a été transférée en totalité la collection Navás. 
Redescription de l'imago $\sigma$ (piquée à sec)

Tête

Face brun translucide moyen à foncé. Vertex brun très foncé. Ocelles grisâtres, pas net tement cerclés à la base. Antennes brun moyen (les deux funicules sont brisés). Yeux bruns, plus foncés dans la région sous-marginale externe; région marginale claire, jaune grisâtre.

\section{Thorax}

Prothorax. Tergite brun assez clair au centre et sur les bords, plus foncé sur les aires latérales en arrière des yeux. Sternite brun foncé uniforme. Pattes 1 brun moyen, sans tache médiofémorale nettement marquée; rapport des longueurs tarse $1 /$ tibia $1=1,48$ (une seule mesure).

Meso et metathorax. Scutum brun moyen brillant, plus clair sur les bords. Mesosternum brun foncé uniforme et teme. Pleures brun moyen à membranes jaunâtre foncé. Scutellum brun foncé brillant mais non pas noirâtre. Pattes 2 et 3 brun jaunâtre, les tarses un peu plus foncés ; aucune trace de tache médiofémorale (les 4 pattes sont encore en place).

Ailes antérieures à membrane hyaline, entière. ment transparente. L'aire ptérostigmatique, claire, légèrement bistre - de même que la moitié distale du champ Sc-R1 - compte une vingtaine de nervures pratiquement pas ramifiées.

Ailes postérieures transparentes à nervation très fine, brun clair.

\section{Abdomen}

Couleur de base des tergites brun brillant foncé à moyen dorsalement, se prolongeant latéralement vers les pleures par une tache incurvée de même couleur sur fond jaune (fig. 1). Sternites plus clairs et surtout plus ternes que les tergites, avec la région centrale brun moyen, les bords postérieurs et latéraux brun jaunâtre.

Cerques absents.

Genitalia

Styligère (fig. 2) un peu plus foncé latéralement. avec deux grandes protubérances assez arrondies. Styles renflés à la base.

Lobes péniens subcylindriques, à apex moins en biseau que chez $R h$. germanica (fig. 3, 4 et 5). Cet apex, de section à peu près circulaire, est totalement dépourvu d'épines ( $f i g .6$ ). Titillateurs larges et multidentés, représentés de face sur la fig. 5 .
Taille

Longueur de l'aile antérieure $\left(\sigma^{\circ}\right): 11,9 \mathrm{~mm}$.

\section{Rhithrogena ryszardi $n ., s p .{ }^{1}$}

Des prospections de la faune benthique de l'oued Tigrigra (affluent de l'oued Sebou, Moyen Atlas marocain) et de ses tributaires par l'un de nous (B.V.) ont permis la récolte de larves de Rhithrogena printanières et de grande taille. Leur élevage a montré qu'il s'agissait d'une espèce nouvelle, voisine des précédentes. Nous sommes heureux de la dédier cordialement à notre ami le Dr Ryszard Sowa, professeur à l'Université Jagiellone (Cracovie), en hommage à ses nombreux travaux sur ce genre d'Ephéméroptères.

Imago o : description

Conservation en alcool à $70^{\circ}$.

\section{Tête}

Face translucide, grise veinée de noirâtre. Vertex brun moyen. Ocelles gris, cerclés à la base d'un large anneau violacé noirâtre. Antennes brun grisâtre assez clair. Yeux gris avec deux bandes latérales externes parallèles violacées, dont une marginale, séparées par une bande gris blanchâtre.

Thorax

\section{Prothorax.}

Tergite jaunâtre dans la zone médiane - avec deux petits points bruns près du bord antérieur et brun foncé sur les côtés, en arrière des yeux. Sternite brun moyen, plus foncé sur le pourtour. Pattes 1 assez courtes, brun très foncé avec une tache médiofémorale noirâtre. Rapport des longueurs tarse 1/tibia 1 faible, compris entre 1,05 et 1,32 pour 10 mesures $(\overline{\mathrm{m}}=1,22)$.

Meso et metathorax.

Scutum brun clair à olivâtre. Mesosternum brun assez foncé. Scutellum brun noirâtre. Pattes 2 et 3 brun clair olivâtre avec une tache violacé noirâtre sur le tiers médian.

Ailes antérieures. Les couleurs décrites ne sont valables que pour des spécimens ayant mué depuis plus de 12 heures. Membrane alaire bistre clair transparent sur toute sa surface ; aire ptérostigmatique et champs S-Sc et Sc-R1 bistre plus foncé. Une

1. Description par A. Thomas et T. Soldan. 

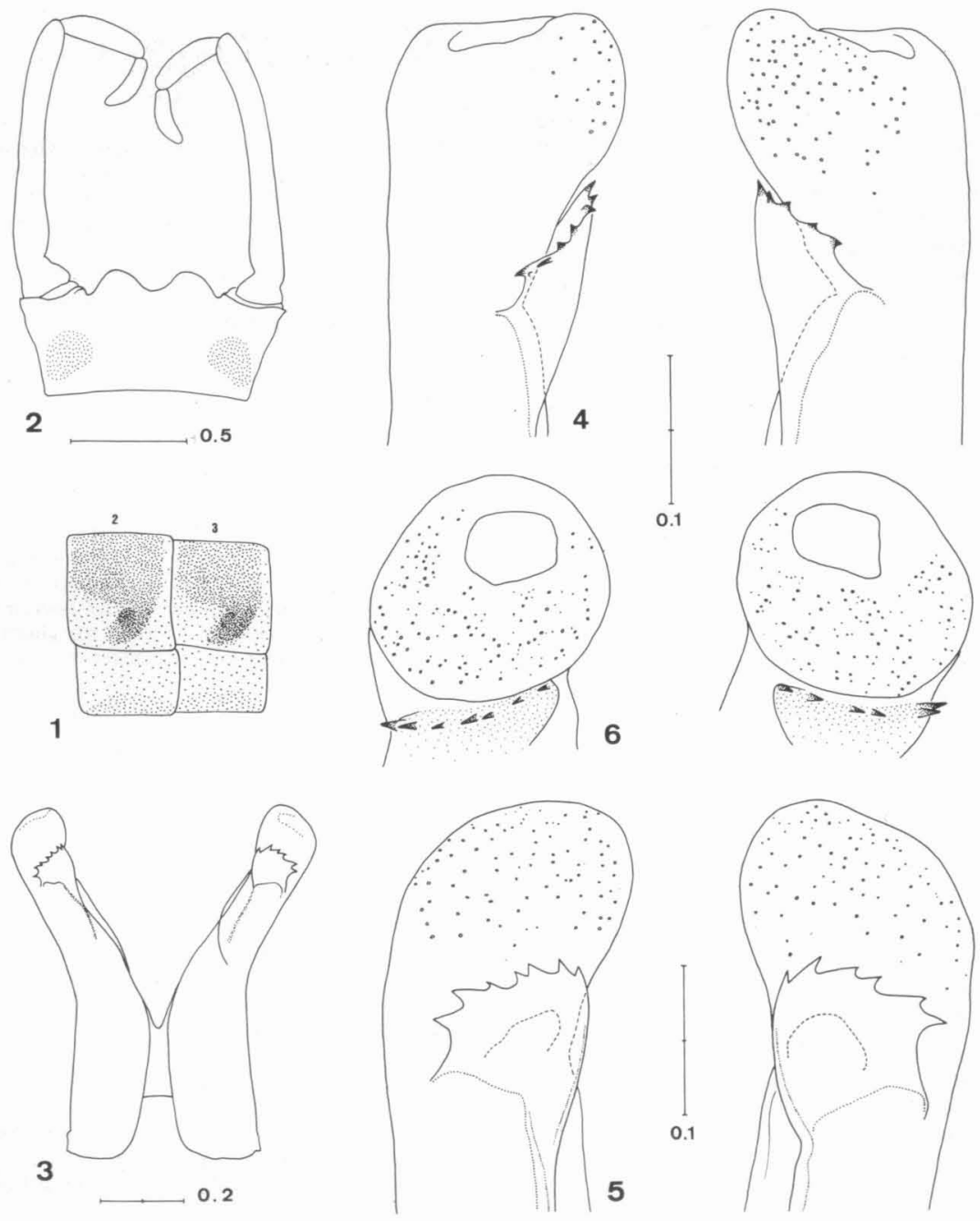

Fig. 1 à 6 . Imago ơ de Rhithrogena soteria Navás. Echelle en mm. $1: 2^{\mathrm{c}}$ et $3^{\mathrm{e}}$ segments abdominaux en vue latérale. 2 : styligère et styles en vue ventrale. 3 : pénis en vue ventrale. 4 à 6 : apex des deux lobes péniens en vues latérale (4), ventrale (5) et caudale (6). 
quinzain£ de nervures transverses dans l'aire ptérostigmatique, non anastomosées et subparallèles. Nervures longitudinales: S, Sc et Rl brun moyen, les autres brun foncé à très foncé. Nervures transverses brun très foncé, soulignées sur la membrane alaire d'un liséré bien marqué entre $C$ et R2, ce sur toute la longueur de l'aile, et aussi entre R2 et MA - parfois même au-delà - mais seulement sur les transverses de la moitié proximale de l'aile. Ce caractère permet de distinguer aisément $R h$. ryszardi des espèces précédentes.

Ailes postérieures hyalines, légèrement bistres à fine nervation longitudinale et transversale brun foncé s'éclaircissant progressivement vers l'arrière.

\section{Abdomen.}

Couleur de base des tergites brun moyen - quelque peu rougeâtre - unj sauf sur les côtés, près des pleures, où deux taches séparées par une zone plus claire peuvent être distinguées, la postérieure plus étendue (fig. 7). Sternites clairs, sauf dans la région centrale brun moyen chez les spécimens ayant mué depuis plusieurs heures. $9^{e}$ sternite brun sur les côtés et la moitié proximale.

Cerques brun foncé.

Genitalia.

Styligère brun moyen, un peu plus foncé latéralement. Deux fortes protubérances séparées par une profonde encoche médiane à bords subrectilignes et largement ouverts (fig. 8).

Styles brun foncé, peu renflés à la base, mais ce caractère est variable.

Lobes péniens (fig. 9 à 12) de morphologie assez proche de ceux de $R h$. soteria, peut être même encore plus nettement tronqués perpendiculairement à l'axe au sommet, et donc bien différents de ceux de $R h$. germanica, en biseau à l'apex. Une épine assez petite mais souvent dédoublée, subapicale, externe et latérodorsale, comme chez cette dernière espèce, est visible, même en vue ventrale. Par rapport à $R$ h. germanica (voir notamment la fig. 1 , p. 487, de Sowa 1971), la faible dépression externe, près de l'épine, est beaucoup plus franchement dorsale (fig. 9a et surtout 9b). La morphologie des genitalia de $R h$. germanica et de $R h$. ryszardi présente toutefois une variabilité certaine, non négligeable, même à l'intérieur d'une seule population. Ce n'est pas la première fois que nous mettons en garde contre cette difficulté chez le genre Rhithrogena (voir par exemple Thomas et Mohati 1985). Titillateurs très larges et multidentés (représentés de face sur la fig. 10), comme chez les deux autres espèces.

\section{Taille}

Longueur de l'aile antérieure: 10,7 à 12,1 mm. Longueur des cerques : 17,5 à $24 \mathrm{~mm}$.

Subimago o (en alcool)

La coloration varie beaucoup selon l'état de déshydratation - et donc l'âge - de l'animal.

Par rapport à l'imago, on relève surtout les différences suivantes:

Coloration plus claire dans l'ensemble, moins brune, tirant davantage sur le jaune ou le gris olivâtre.

Yeux dépourvus de bandes latérales nettes, la région marginale externe seulement plus foncée que le reste.

Thorax jaunâtre dorsalement, latéralement et ventralement, avec quelques marques brun foncé localisées aux régions antérieure et latérales du scutum. Mesosternum jaunâtre avec liséré latéral brun noirâtre. Une tache noirâtre sur les pleures, dans le prolongement de Sc.

Pattes olivâtres à tache médiofémorale petite mais bien contrastée et tarses assombris.

Ailes antérieures et postérieures enfumées, à nervures longitudinales claires et transverses très assombries par des lisérés de pigment violacé.

Abdomen à taches latérales pluttôt plus apparentes que chez l'imago en raison des tergites brun clair.

Cerques brun foncé : longueur : 13 à 14,5 mm.

Genitalia (fig. 13).

Protubérances du styligère très rapprochées et très saillantes, arrondies, séparées par une encoche étroite et profonde.

Lobes péniens plus convergents que chez l'imago.

Matériel examiné.

10 individus $\sigma$ (B. Vitte leg.) obtenus par élevage et provenant de deux oueds tributaires de l'o. Tigrigra (Moyen Atlas marocain) :

- 5 i. et 3 subimagos, o. Tout à $1260 \mathrm{~m}$ (localité typique), mai 1986;

- $2 \mathrm{i}$., o. Ben Çmim à $1260 \mathrm{~m}$, mai 1985.

Un paratype est déposé au Musée Zoologique de Lausanne, un autre dans la collection Soldán à Ceske Budejovice, l'holotype et le reste du matériel dans la collection Thomas à Toulouse. 

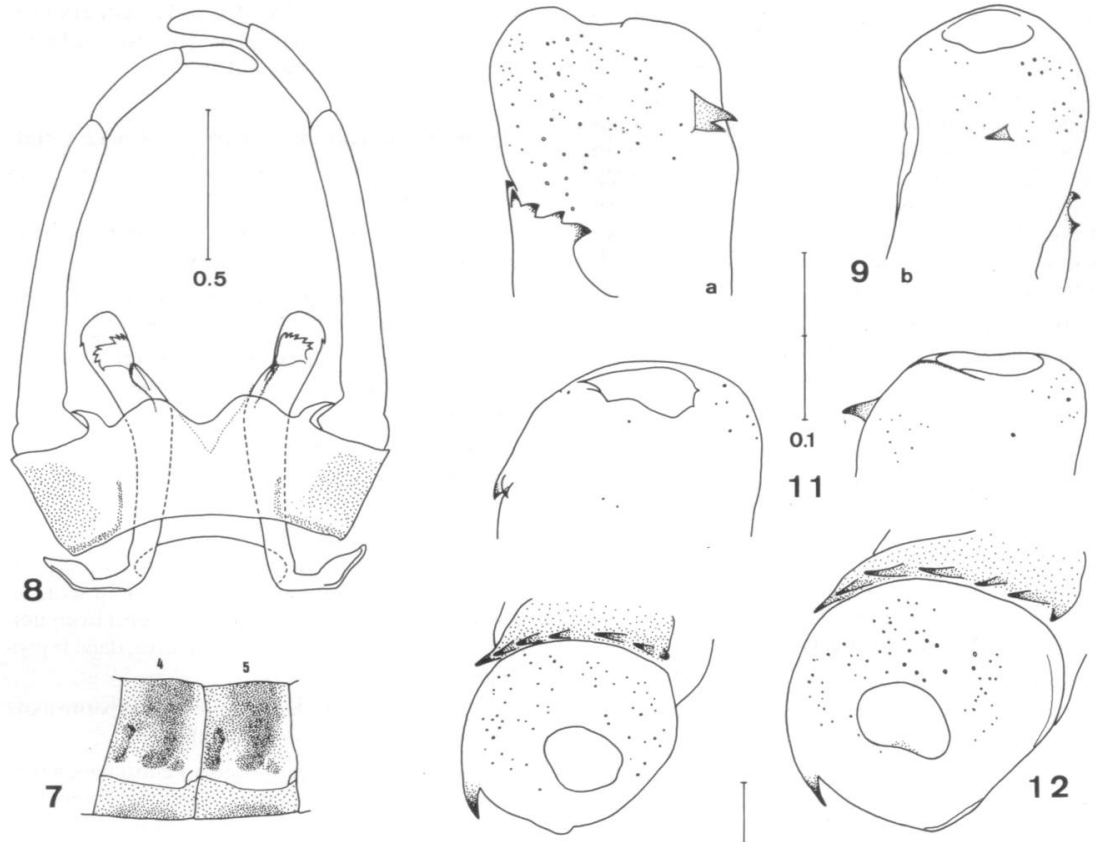

11
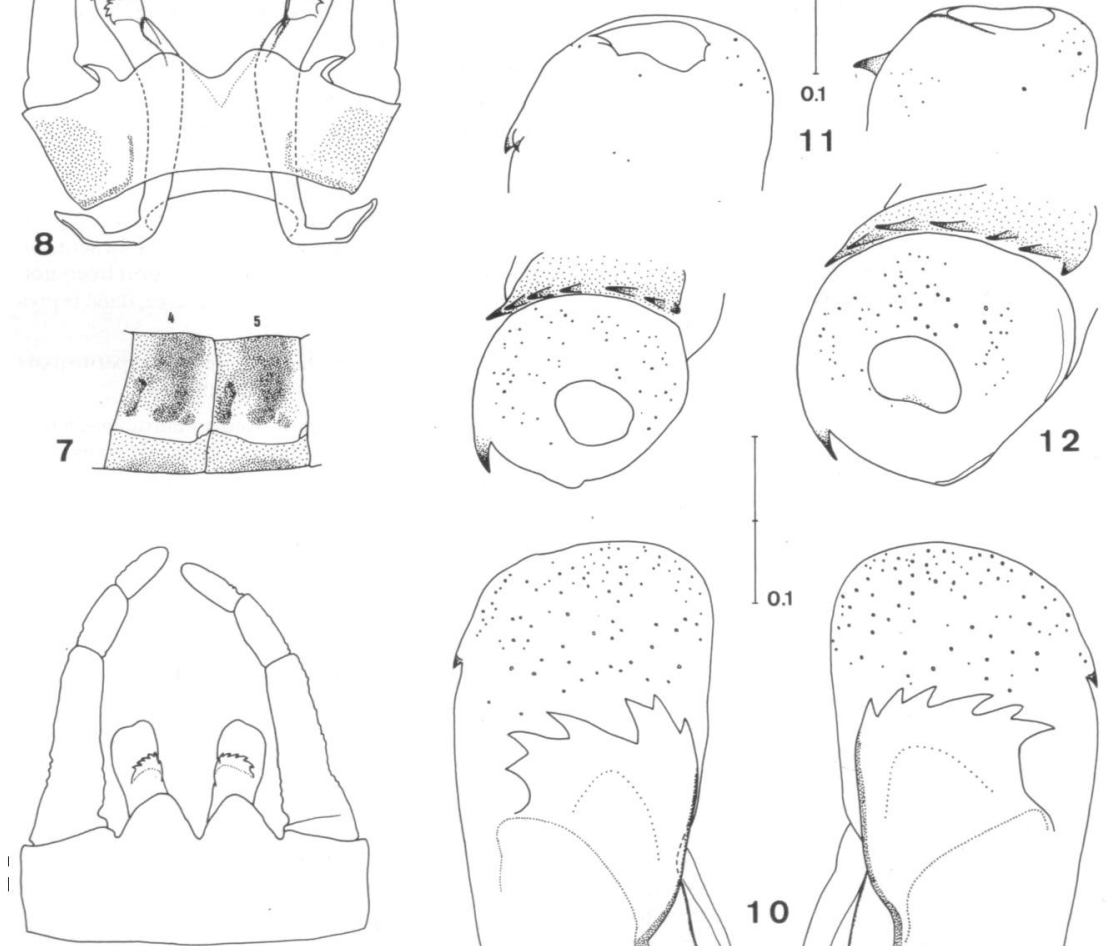

13 0.5

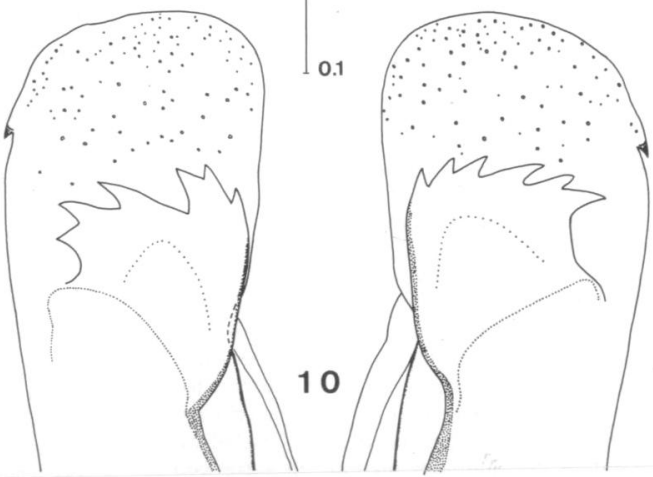

Fig. 7 à 13. Imago $\sigma$ de Rhithrogena ryszardi $\mathrm{n}$. sp. Echelle en $\mathrm{mm} .7: 4^{e}$ et $5^{\text {e }}$ segments abdominaux en vue latérale. 8 ; ensemble des genitalia en vue ventrale. 9 à 12 : lobes péniens de plusieurs spécimens en vues latérale $(9 \mathrm{~b}$ légèrement plus dorsale que 9 a), ventrale (10), dorsale (11) et caudale (12). 13 : genitalia de subimago $O$ en vue ventrale. 


\section{Ecologie (données B. Vitte)}

A la station typique (zone volcanique d'Azrou), l'oued Tout coule dans un lit encaissé. Une grande partie du fond est stable, constituée de blocs et de roche en place, biotope à $R h$. ryszardi. De décembre à mars, le régime des eaux est torrentiel (courant fort, largeur moyenne supérieure à un mètre, profondeur moyenne supérieure à $0.5 \mathrm{~m}$ ). D'avril à juin, le débit et la vitesse diminuent dans de fortes proportions. A partir de juillet, le cours d'eau s'assèche progressivement jusqu'en octobre : seules subsistent pendant l'été quelques zones à courant très lent et des portions stagnantes. La largeur moyenne est alor's de 20 à $30 \mathrm{~cm}$, la hauteur d'eau de quelques $\mathrm{cm}$ seulement (observations relatives aux années 1985 et 86 ).

Pendant la période de vol, en mai, la température diurne maximale de l'eau est de $15 \cdot 16^{\circ} \mathrm{C}$. A la miseptembre, elle est de l'ordre de $18^{\circ} \mathrm{C}\left(22^{\circ} \mathrm{C}\right.$ enregistrés dans les zones stagnantes).

Le pH est compris entre 7,4 et 7,8 .

Nous formulerons donc deux remarques à propos de l'écologie et de la biologie de $R h$. ryszardi :

- le déroulement de son cycle de développement est tel que les larves moyennes et âgées peuvent vivre en courant rapide. En élevage, cette espèce se montre assez fragile, exigeant des eaux agitées et saturées en oxygène. La transformation de la subjmago en imago est longue, de l'ordre de 3 à 4 jours en lumière atténuée (laboratoire). Des études ultérieures sont nécessaires pour préciser si l'été est franchi par l'espèce sous forme d'œufs ou de larvules.

- elle apparaît inféodée au rhithral alors que $R h$. germanica et très probablement $R h$. soteria sont caractéristiques de l'hyporhithral et surtout de l'épipotamal. Il s'agit sans doute là d'une adaptation rendue nécessaire par les concentrations en sels dissous, souvent très élevées, du cours inférieur de nombreux oueds nord-africains, beaucoup d'espèces benthiques restant alors cantonnées en amont (Boumaiza et Thomas 1986).

\section{Les Rhithrogena d'Afrique du Nord}

Le genre Rhithrogena a été cité d'Afrique du Nord pour la première fois par Eaton (1899) d'après la récolte d'un unique spécimen provenant de Biskra (Algérie).

En réalité, ce genre est présent sur tout le Maghreb puisque nous avons pu examiner des exemplaires - le plus souvent des larves - provenant du Haut At las et du Moyen Atlas (Maroc), des monts de Tlemcen et de Grande Kabylie (Algérie), enfin de Kroumirie (Tunisie).

Depuis 1985, 4 espèces nord-africaines ont été décrites, toutes du Maroc. Les trois premières paraissent être des endémiques très alticoles. $R h$. ryszardi, moins orophile, possède probablement une aire de répartition beaucoup plus étendue vers l'Est.

Voici un rappel et quelques compléments concernant la répartition (carte 2 ) et les séries types de ces espèces :

\section{Rhithrogena ayadi Dakki et Thomas, 1986.}

Localité typique: la source Amane Imelallen, alt. 2150 m. Massif montagneux du Bou Iblane, sur le flanc NW, bassin de l'oued Zloul, à environ $3 \mathrm{~km}$ à l'E du refuge de Taffert (Moyen Atlas) et $320 \mathrm{~km}$ à l'E de Casablanca. Juillet 1982.

Holotype et deux paratypes or dans la collection Thomas. D'autres spécimens dans les collections du labora. toire de Zoologie de l'Institut Scientifique de Rabat.

2. Rhithrogena giudicelliorum Thomas et Bouzidi, 1986.

Localité typique : l'assif N'Ouarzane au pied des cascades d'Irhoulidene, alt. $2800 \mathrm{~m}$, vallée d'Ouassadene. Massif montagneux du Toubkal (Haut Atlas), à $60 \mathrm{~km}$ au S de Marrakech. Récolte du 21.VII. 1985

8 imagos $\sigma$. Holotype et 5 paratypes dans la collection Thomas. Un paratype dans les collections du laboratoire d'Hydrobiologie de l'Université Caddi Ayyad (Marrakech) et un autre à l'Institut Scientifique (Rabat).

\section{Rhithrogena ourika Thomas et Mohati, 1985.}

Localité typique : l'oued Ourika à Setti Fadma, alt. 1500 $\mathrm{m}$, à $17 \mathrm{~km}$ à l'ENE du sommet de l'Oukaimeden. Autre station proche : l'assif Tiferguine à $2620 \mathrm{~m}$. Haut Atlas, respectivement à 55 et $60 \mathrm{~km}$ au SSE de Marrakech. Récoltes du 12.VI.1984

24 imagos o. Deux paratypes déposés au Musée Zoologique de Lausanne, l'holotype et plusieurs paratypes dans la collection Thomas (Toulouse) ; d'autres exemplaires au laboratoire d'Hydrobiologie de Marrakech et à l'Institut Scientifique de Rabat.

\section{Rhithrogena ryszardi n. sp.}

Voir $\$ 1,3$. 


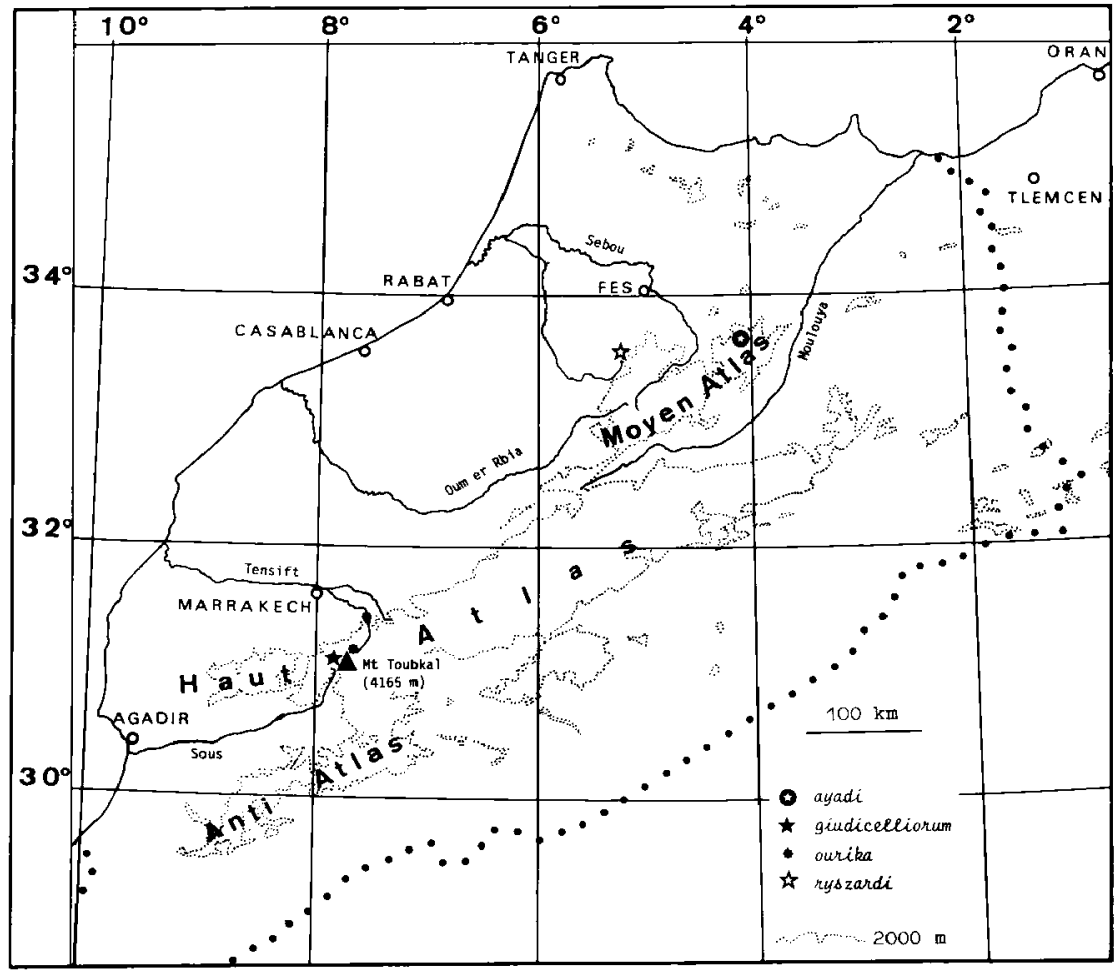

Carte 2 ; Répartition des espèces de $R$ hithrogena nord-africaines actuellement identifiées

\section{Conclusion et perspectives d'avenir}

Trois espèces de Rhithrogena, très voisines et vicariantes, constituent le groupe germanica en Europe de l'Ouest et en Afrique du Nord. Les deux premières, Rh. germanica Eaton et $R h$. soteria Navás, sont potamophiles d'eaux rapides. Pour cette raison, leur survie apparaît actuellement très incertaine.

$R h$. soteria en particulier, endémique espagnole sans doute déjà rare au temps de Navás, n'a pas, à notre connaissance, été retrouvée depuis (AlbaTercedor 1981). Il est possible qu'elle ait disparu du bassin de l'Ebre en raison des perturbations hydrauliques entraînées par l'édification de plusieurs barrages. Sa redécouverte serait très intéressante.

Rh. ryszardi n. sp. est moins directement menacée, parce que capable de coloniser le rhithral, sensiblement moins exposé aux influences humaines, surtout en Afrique du Nord. 


\section{Remerciements}

Nous avons plaisir à remercier pour nous avoir prêté, sur une longue durée, le type de $R h$. soteria et un spécimen de Rh. germanica de Suisse : M. le Révérend Père Palazon Delattre (Saragosse) et M. le conservateur H.D. Volkart (Berne).

\section{Travaux cité:}

Alba-Tercedor (J.). 1981. - Recopilacion de citas de Efemeropteros en la peninsula iberica e is las Baleares. Trab. Monogr. Dep. Zool. Univ. Granada, (N.S.), 4.(2): $41-81$.

Alba-Tercedor (J.) et Sowa (R.). 1986. - Two interesting Rhithrogena Eaton from Spain : $R$. thomasi $\mathrm{sp}$. $\mathrm{n}$., and $R$. monserrati sp. n. (Ephemeroptera : Heptageniidae). Aquatic Insects, 8 (3): 185-189.

Belfiore (C.). 1983. - 24. Efemerotteri (Ephemeroptera). In : Guide per il riconoscimente delle specie animali delle acque inteme italiane. Stamperia Valdonega, $113 \mathrm{p}$. Verona.

Boumaiza (M.) et Thomas (A.G.B.). 1986. - Répartition et écologie des Ephémėroptères de Tunisie (tere partie) (Insecta, Ephemeroptera). Archs Inst. Pastetur Tinis, 63 (4) ; 567-599.

Dakki (M.) et Thomas (A.G.B.). 1986. - Rhih rogena ayadi n. sp., Ephéméroptère nouveau du Moyen Atlas marocain (Heptageniidae). Annls Limnol., 22 (1) : 27-29.

Despax (R.). 1949. - Addition à la faune des Ephéméroptères de France : Rhithrogena haarupi dans les Pyrénées. Bull. Soc. Hist. nat. Toulouse, 84 (3-4) : 145-146.

Eaton (A.E.). 1883-88. - A revisional monograph of recent Ephemeridae of Mayflies. Trans. Linn. Soc, 3 (Zoology) : 1.352
Eaton (A.E.). 1899. - List of Ephemeridae hither to observed in Algeria with localities. Entomologist's mon. Mag. 35 : 45

Macan (T.T.). 1979. - A key to the nymphs of British Ephemerop tera. F.B.A. Scient. Publ. $n^{\circ} 20: 1.80$.

Navás (L.). 1917. - Neuropteros nuevos o poco conocidos ( 9 a serie). Mem. R. Acad. C. Ant. Barcelona, 13 (26): 393-406.

Puig (M.A.). 1980. - Contribució a l'estudi de l'ecologia compa rada dels Plecopters i Efemeropters d'Andorra. Butll, Inst, cal. Hist. nat... 45 (Sec. Zool., 3) : 77-87.

Puthz (V.). 1978. - Ephemeroptera. In J. Illies : Limnofauna Euro paea. Fischer Verlag. Stuttgart : 256-263

Sowa ( $R$.) 1971. - Note sur quelques Rhithrogena Eaton de la collection Esben-Petersen et la redescription de Rhithrogena germanica Eaton (Ephemeroptera, Heptageniidae). Bull. Acad, pol. Sci. (Ser. Sci. biol.), 19 (7-8) : 485-492.

Sowa (R.). 1975, - Ecology and biogeography of mayflies (Ephe meroptera) of running waters of the Polish part of the Carpathians. 1. Distribution and quantitative analysjs. Acta Hydro biol., 17 (3) : 223-297.

Thomas (A.). 1968. - Quelques Ecdyonurus et Rhithrogena européens de la collection Navás (Ephemeroptera). Annls Limnol., $4(2): 209-218$

Thomas (A.G.B.) et Bouzidi (A.). 1986. - Trois Ephéméroptères nouveaux du Haut Atlas marocain (Heptageniidae, Baetidae, Leptophlebiidae). Bull. Soc. Hist, nat. Toulouse, 122 : 7-11.

Thomas (A.G.B.) et Mohati (A.). 1985. - Rhithrogena ourika n. sp. Ephéméroptere nuuveau du Haut Atlas marocain (Heptageniidae). Amnts Limnol., 21 (2): 145-148.

Thomas (A.G.B.) et Sartori (M.). 1985. - Rhithrogena gorrizi Navás, 1913 et $R h$. castellana Navás, 1927 : redescription des imagos (Ephemeroptera, Heptageniidae). Annls Limnol, 21 (1) $65-70$ 